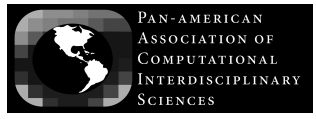

Journal of Computational Interdisciplinary Sciences (2009) 1(2): 113-117

(C) 2009 Pan-American Association of Computational Interdisciplinary Sciences

ISSN 1983-8409

http://epacis.org

\title{
A numerical study for non-stationary anomalous diffusion considering non-inversive velocity correlation functions
}

\author{
Marcel V.S. Santos ${ }^{1}$, Cássia C. Donato ${ }^{1}$, Ismael V.L. Costa ${ }^{2}$ and Fernando A. Oliveira ${ }^{1}$ \\ Manuscript received on December 12, 2008 / accepted on January 12, 2009
}

\begin{abstract}
In this work we used a previous theory [R. Morgado, F.A. Oliveira, G.G. Batrouni, and A. Hansen, Phys. Rev. Lett. 89, 100601 (2002)] to explain and resolve the non-stationary diffusive problem studied by Srokowski [T.E. Srokowski, Phys. Rev. Lett. 85, 2232 (2000); Phys. Rev. E 64, 31102 (2001)]. In order to do that, we have used the generalized Langevin's formalism to develop a computational algorithm and treat the problem numerically. To confirm the result of the method, an analytical alternative utilizing the final value theorem for Laplace transforms was derived and both results were compared. After all, it was presented a final characterization to the anomalous diffusive system in question through the calculation of some parameters like correlation function, diffusion coefficient and mean square displacement.
\end{abstract}

Keywords: Anomalous diffusion, memory function, correlation function.

${ }^{1}$ Instituto de Física and Centro Internacional de Física da Matéria Condensada, Universidade de Brasília, Caixa Postal 04513, 70919-970 Brasília, DF, Brazil.

2Universidade Federal de Goiás, Campus Jataí, 75800-000 Jataí, Goiás, Brazil. 


\section{INTRODUCTION}

Diffusion is one of the fundamental mechanisms for transportation of energy, mass and information. In many physical systems, this process is the main way to achieve the equilibrium and it is worth mention that this issue has been the focus of extensive research in various branches of science. However, there are still many open questions concerning this matter, especially in systems which are outside the state of equilibrium or those in which it is verified the existence of anomalous diffusion [1].

The study of systems with memory reveals some physical phenomena that are still not well understood. In this paper, we studied a problem in anomalous diffusion proposed by Srokowski [2]. In his work, Srokowski studied a Brownian motion, which presented a anomalous dynamic due to a memory function given by Eq. (14). Comment on this work have been done [3], however no solution have been presented up to now for this problem. Our objective in this work is to present a clear description of the problem. In order to do that, we derived a numerical method to obtain the correlation function of velocities for an ensemble of particles from any given memory as long as the diffusive process in question can be described by a Generalized Langevin Equation (GLE).

The structure of the paper is constructed as the following: in Section 2 we will present an analytical way of getting a correlation function from a memory function; in Section 3 we describe in details the numerical approach developed to obtain the response function; in Section 4 the methods proposed to will be applied to the diffusive system studied by Srokowski [2]; and finally, in Section 5 there will be a brief summary of the main results obtained.

\section{GENERALIZED LANGEVIN EQUATION AND DIFFUSION}

The GLE is a stochastic differential equation which can be used to model systems driven by colored random forces. Those kind of systems are not unusual and it is possible to find them in many areas, such as the dynamics of dipolar [4], polymeric chains $[5,6,7,8,9]$, metallic liquids [10], Lennard Jones liquids [11], diffusion in periodic potentials [12], ratchet devices [13, 14], and synchronization [15, 16, 17].

This equation can be written for a given operator $A(t)$ as:

$$
\frac{d A(t)}{d t}=-\int_{0}^{t} \Gamma\left(t-t^{\prime}\right) A\left(t^{\prime}\right) d t^{\prime}+\xi(t),
$$

where $\Gamma(t)$ is the retarded friction kernel of the system, or the memory function. Here, $\xi(t)$ is a stochastic noise subject to the conditions $\langle\xi(t)\rangle=0,\langle\xi(t) A(0)\rangle=0$, and

$$
C_{\xi}(t)=\langle\xi(t) \xi(0)\rangle=\left\langle A^{2}(t)\right\rangle \Gamma(t),
$$

where $C_{\xi}(t)$ is the correlation function for $\xi(t)$ and the angular brackets denote an average over the ensemble of particles. Equation (2) is the famous Kubo's Fluctuation Dissipation Theorem (FDT) [18] and is quite general.

The GLE models the non-Markovian movement of a particle with mass $m$ moving up in a fluid of time dependent viscosity $\Gamma(t)$, subject to a force of friction proportional to the speed (first term on the right side, connected to the dissipation) and a random force in time (second term, connected to the fluctuation). The random force stems from the impact with other particles of the fluid in which it is submerged. Moreover, the presence of the kernel $\Gamma(t)$ allows us to study a large numbers of correlated processes. In the real world, the vast majorities of problems are nonMarkovian, i.e., there is correlation between the various stages of dynamic evolution, and thus the particles are able to do a next action based on any previous history. This property is what we call memory and it makes remote events of the past important to dynamic events in present.

Notice that if $A(t)$ is the momentum of a particle with unit mass,

$$
x(t)=\int A(t) d t,
$$

will be its position. Using this definition it is possible to study the asymptotic behaviour of its second moment,

$$
\lim _{t \rightarrow \infty}\left\langle x^{2}(t)\right\rangle \sim t^{\alpha},
$$

to characterize the type of diffusion presented by the system. For $\alpha=1$ we have normal diffusion and $\lim _{t \rightarrow \infty} D(t)=$ constant. Here, $D(t)$ is the diffusion coefficient as a function of time. For $\alpha \neq 1$ we have anomalous diffusion: if $\alpha<1$ then $\lim _{t \rightarrow \infty} D(t)=0$ and the motion is subdiffusive; if $\alpha>1, \lim _{t \rightarrow \infty} D(t)=\infty$, and the motion is superdiffusive. Equation (4) is a generalization of Einstein's relation for the mean square displacement of an ensemble of particles and it will be widely used in this work.

To characterize the diffusion phenomena, it will be discussed the behaviour of the correlation function

$$
R(t)=\frac{\langle A(t) A(0)\rangle}{\langle A(0) A(0)\rangle}
$$

from which we can describe most of the diffusion processes of interest. This function is normalized to enable control of the initial value of it when numerical calculus is needed. 
Using the previous conditions for the noise, it is possible to obtain a self-consistent equation for $R(t)$. It can be made multiplying both sides of Eq. (1) by $A(0)$ and taking the average over the ensemble. This equation is given by

$$
\dot{R}(t)=-\int_{0}^{t} \Gamma\left(t-t^{\prime}\right) R\left(t^{\prime}\right) d t^{\prime}
$$

and it is important because it can be resolved numerically in order to get the correlation function, diffusion coefficient and the mean square displacement. Those parameter are the main way to characterize a diffusion. If we then apply the Laplace transform to Eq. (6), we get

$$
\widetilde{R}(z)=\frac{1}{z+\widetilde{\Gamma}(z)} .
$$

It is possible to obtain plenty of information concerning the asymptotic behaviour of the system $[1,18,19,20]$, taking the Laplace transform of the correlation function through the memory function of the system. Unfortunately, in some cases it is not so easy to inverse this transform. In those situations, using numerical methods is a good alternative to overcome this problem.

Despite the difficulty of getting the exact analytical equation of the correlation function from a given memory, there is a way to calculate its final value, using the final value theorem (FVT) for Laplace transforms, which is

$$
\lim _{t \rightarrow \infty} D(t)=\lim _{z \rightarrow 0} z \widetilde{D}(z)
$$

If we combine this equation with the Kubo's definition for the diffusion constant [21],

$$
D(t) \sim \int_{0}^{t} R(t) d t
$$

we get the equality

$$
\lim _{t \rightarrow \infty} D(t)=\lim _{z \rightarrow 0} \widetilde{R}(z) .
$$

This equation is very powerful. It gives the final value of the diffusion coefficient, using just the Laplace transform of the memory function as input in Eq. (7). This kind of approach is important to verify the solution obtained with the numerical method, since it is a very strong form of getting an analytical characterization for any diffusive system.

\section{NUMERICAL APPROACH}

In order to describe diffusive systems with memory that implies non-inversive correlation functions, it is possible to calculate
Eq. (6) numerically. To do this, firstly it is necessary to rewrite this equation into a discrete form. From Eq. (1) we obtain

$$
\begin{gathered}
R(t+\Delta t)= \\
=-\Delta t \int_{0}^{(t+\Delta t)} \Gamma\left(t+\Delta t-t^{\prime}\right) R\left(t^{\prime}\right) d t^{\prime}+R(t) .
\end{gathered}
$$

This expression is not so easy to manipulate. To put it into a more clear way, it is possible to resolve part of the integral above and then to expand it until the second order term in $\Delta t$ to obtain

$$
R(t+\Delta t)=\frac{(1-\sigma)}{(1+\sigma)} R(t)-\frac{\Delta t}{(1+\sigma)} I(t),
$$

where $\sigma=\Gamma(0) \Delta t^{2} / 2$, and $I(t)$ is given by

$$
I(t)=\int_{0}^{t} \Gamma\left(t-t^{\prime}\right) R\left(t^{\prime}\right) d t^{\prime} .
$$

The convolution integral above is a very important part of Eq. (12), and the numerical algorithm will spent most of time calculating its values for every increment $\Delta t$. This iterated function is the basis of the algorithm which generates the numerical values of the correlation function of velocities for an ensemble of particles with a given memory. From these values, its possible to get the diffusion coefficient through Eq. (9), and then one can characterize completely the type of diffusion of any diffusive system, i.e. one can determine the diffusive exponent $\alpha$.

\section{RESULTS}

At this point, it is interesting to apply the presented numerical method to analyze the memory function proposed by Srokowski [2], which is a two-step retarded friction kernel written as

$$
\Gamma\left(t-t^{\prime}\right)=\left\{\begin{array}{ll}
1 / \varepsilon & t \leq \varepsilon \\
1 / t & t>\varepsilon
\end{array},\right.
$$

where $\varepsilon$ is an arbitrary small number. For this kernel, the author studied both the formal solution and a Monte Carlo method known as the Kangaroo process. He computes the velocity correlation function and concludes that the diffusion constant is finite for normal solution and infinite for the Kangaroo process. It means that the diffusion of a system with the memory proposed should be characterized as normal or superdiffusive. Even though Srokowski's methods sound interesting, his results for diffusion constant are however not appropriate.

It is well known that long range correlation functions may induce anomalous behaviour, such as delayed fracture [5, 7], metastability in nucleation [6, 22], and anomalous reaction rate [23]. However, it may not induce infinite diffusion where it does not exist or an unrealistic constant as in the present case. 
Analyzing the process with the analytical development presented in previous sections, we can take the Laplace transform of the memory function exposed in Eq. (14). Thus, we obtain

$$
\widetilde{\Gamma}(z)=\frac{1-e^{-\varepsilon z}}{\varepsilon z}+E i(\varepsilon z),
$$

with the integral exponential function $E i(\varepsilon z)$ defined [24] as the series:

$$
E i(x)=\gamma+\ln (x)+\sum_{n=1}^{\infty} \frac{x^{n}}{n ! n},
$$

where $\gamma$ is the Euler-Mascheroni constant.

Using Eq. (7) and (10) it is possible to obtain the analytical result of the diffusion coefficient for the system in discussion. For that, we use $z \rightarrow 1 / t, \epsilon=0.01$, and Eq. (15) for values of $x<0.01$. So, we have

$$
D(t) \sim \frac{1}{k+\ln (t)},
$$

where $k$ is a constant given by $k=1+E i(0.01)=5.0379$.

Equation (17) is the final form of the decaying curve for the diffusion coefficient. So, we can just compare some other result with this one in a certain scale of time which is long enough to be approximated as infinite or when the system analyzed has already entered in the stationary regime. Now, if we apply the limit $\lim _{t \rightarrow \infty} D(t)$ to Eq. (17), we find that the diffusion coefficient goes to zero as the time goes to infinite. This limit characterizes the subdiffusive anomalous diffusion and it goes against the result found by Srokowski. To confirm the analytical result derived here, we applied the numerical method developed for the memory in question.

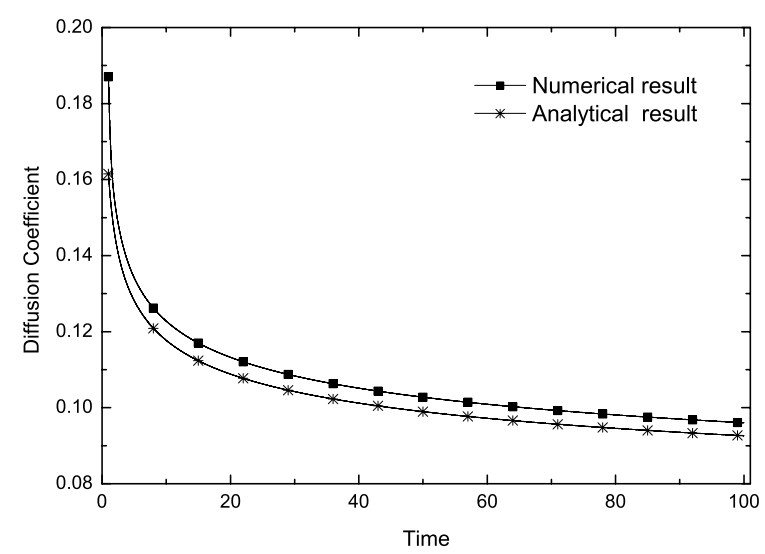

Figure 1 - Diffusion coefficient as a function of time. The upper curve is the numerical result while the lower one is obtained through the FVT. A time scaling in the form $t^{\prime}=\lambda t$ can transform one curve in the other.
In Figure 1 we plot the diffusion coefficient $D(t)$ as a function of time. The curve corresponding to numerical development was calculated with Eq. (12), for $\varepsilon=0.01, \Delta t=10^{-5}$. It is important to mention that to use the FVT correctly, it is necessary to explicit the decaying form of the curve in question. The Laplace transform decays, for this case, with a law given by $z \rightarrow 1 / t$. It is a nice surprise that the FVT agrees with the numerical calculation, since it is a theorem only for the final result. We fit the numerical results using the Eq. (17) to obtain the numerical value $k$ num $=5.8188$. The difference between the numerical and analytical results is an important point about the method, indeed the FVT works both for $z=1 / t$ or $z=\lambda / t$, where $\lambda$ is a constant. Unfortunately only the numerical calculation will provide us $\lambda$. Now, if we use $z=\lambda / t$ and we derive again Eq. (17) we get $k-\ln \lambda=k_{\text {num }}$ or $\lambda=0.46$, with that correction we get the right analytical limit, i.e. both curves colapse into a single one.

In Figure 2 we display the mean square displacement < $x^{2}(t)>$ as function of time $t$. After the small range of transient regime, the diffusion appears to be normal, since it has a very slow decaying of the diffusion constant and the curve appears to be a line. However, the $\ln (t)$ in $\mathrm{Eq}$. (17) will make the motion subdiffusive in all range studied, because the limit of this function is zero when the time goes to infinite. Despite the difficult of characterize this case as a usual power law, it was made a nonlinear fitting of the curve in the interval analyzed. The result of this proceeding was a diffusion exponent $\alpha \sim 0.9$. It means that according with this other approach, the system is still subdiffusive, and it confirms our result one more time. This somehow shows the limit of fitting, since it is very difficult that the $\ln (t)$ will appear without some previous theory.

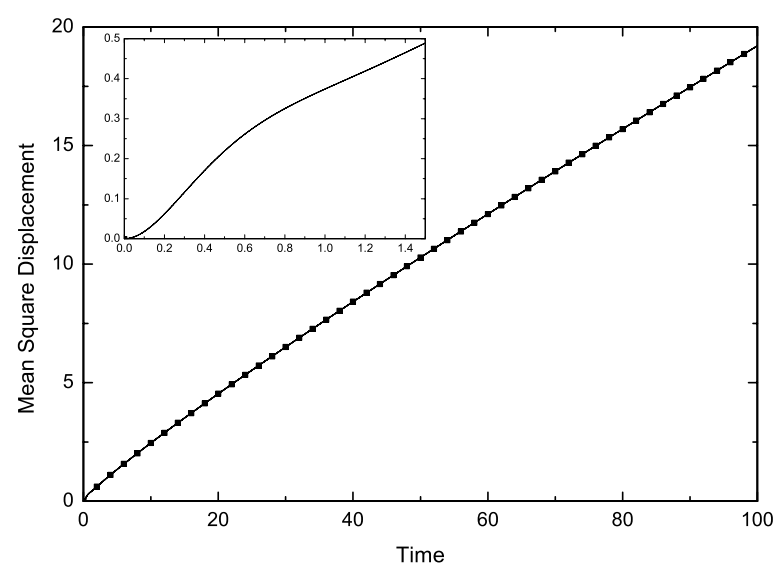

Figure 2 - Mean square displacement as a function of time. After the transient time, which is shown in detail, the curve displays a subdiffusive process. 


\section{CONCLUDING REMARKS}

In this work we studied the non-stationary diffusive system proposed by Srokowski [2]. He had obtained by numerical integration a superdiffusion, while using the Kangaroo process he obtained a normal diffusion. In this work, it was developed a numerical method to analyze some anomalous diffusive processes which have non-inversive correlation function of velocities. The final value theorem for Laplace transforms was also utilized to construct an alternative way to treat problems like this one, for the asymptotic behavior. After all, the results from both methods display a subdiffusive motion, which is a very different result of that of Srokowski. We compare as well the result of the diffusive coefficient $D(t)$ for large times, and it was found the time scaling factor $(\lambda)$ that adjusts both results. I.e. if we use the scale transformation $t^{\prime}=\lambda t$, it corrects the asymptotic result of the Laplace transform, and the numerical and analytical results collapse into one.

It was also verified that the final value theorem agreed with a very great accuracy with the numerical calculation proposed. This is an important and surprising result because the FVT is rigorously valid only in a infinite time. In this way, we overcame an old problem existing in literature. An essential concluding remark that should be done is that diffusion is still a modern field of research and it involves problems such as dispersion of crumpled wires [25], the validation of the fluctuation-dissipation theorem [18], general properties of the correlation function [19], ergodic hypothesis [19], entropy [20], and the Khinchin theorem [26]. The research of correlated phenomena, such as growth, which is the dispersion of a height with specific scaling rules [24], will be improved with a better understand of diffusion.

\section{ACKNOWLEDGMENTS}

This work was supported by CNPq, CAPES, and FAP/DF.

\section{REFERENCES}

[1] MORGADO R, OLIVEIRA FA, BATROUNI GG \& HANSEN A. 2002. Phys. Rev. Lett., 89: 100601.

[2] SROKOWSKI TE. 2001. Phys. Rev. Lett., 85: 2232; Phys. Rev. E, 64: 31102.

[3] OLIVEIRA FA, MORGADO R, DIAS CL, BATROUNI GG \& HANSEN A. 2001. Phys. Rev. Lett., 86: 5839

[4] TOUSSAINT R, HELGESEN G \& FLEKKØY EG. 2004. Phys. Rev. Lett., 93: 108304.
[5] OLIVEIRA FA \& TAYLOR PL. 1994. J. Chem. Phys., 101: 10118

[6] OLIVEIRA FA \& GONZALEZ JA. 1996. Phys. Rev. B, 54: 3954.

[7] OLIVEIRA FA. Phys. Rev. B, 57: 10576 (1998).

[8] MAROJA AM, OLIVEIRA FA, CIESLA M \& LONGA L. 2001. Phys. Rev. E, 63: 061801.

[9] DIAS CL, DUBE M, OLIVEIRA FA \& GRANT M. 2005. Phys. Rev. E, 72: 011918

[10] RAHMAN A, SINGWI KS \& SJÖLANDER A. 1962. Phys. Rev., 126: 997.

[11] YULMETYEV FM, MOKSHIN AV \& HANGGI P. 2003. Phys. Rev. E, 68: 051201.

[12] SANCHO JM, LACASTA AM, LINDENBERG K, SOKOLOV IM \& ROMERO AH. 2004. Phys. Rev. Lett., 92: 250601.

[13] BA0 JD \& ZHUO YZ. 2003. Phys. Rev. Lett., 91: 138104.

[14] BAO JD, ZHUO YZ, OLIVEIRA FA \& HANNGI P. 2006. Phys. Rev. E, 74: 061111

[15] LONGA L, CURADO EMF \& OLIVEIRA FA. 1996. Phys. Rev. E, 54: 2201(R)

[16] CIESLA M, DIAS SP, LONGA L \& OLIVEIRA FA. 2001. Phys. Rev. E, 63: 065202(R).

[17] MORGADO M, CIESLA M, LONGA L \& OLIVEIRA FA. 2007. EPL, 79: 10002

[18] COSTA IVL, MORGADO R, LIMA MVBT \& OLIVEIRA FA. 2003. EPL, 63: 173.

[19] VAINSTEIN MH, COSTA IVL, MORGADO R \& OLIVEIRA FA. 2006. EPL, 73: 726.

[20] LAPAS LC, COSTA IVL, VAINSTEIN MH \& OLIVEIRA FA. 2007. EPL, 77: 37004

[21] KUBO R. 1966. Rep. Prog. Phys., 29: 255.

[22] GONZALEZ JA \& OLIVEIRA FA. 1999. Phys. Rev. B, 59: 6101.

[23] OLIVEIRA FA. 1998. Physica A, 257: 128

[24] MELLO BA, CHAVES AS \& OLIVEIRA FA. 2001. Phys. Rev. E, 63: 041113.

[25] DONATO CC, OLIVEIRA FA \& GOMES MAF. 2006. Physica A, 368: 1.

[26] LAPAS LC, MORGADO R, VAINTEIN MH, RUBÍ JM \& OLIVEIRA FA. 2008. Phys. Rev. Lett., 101: 230602. 